\title{
Acquired surface dysgraphia and dyslexia in the semantic variant of primary progressive aphasia: a single-case study in Spanish
}

\section{Autores}

Morello García Florentina, Difalcis Micaela, Leiva Samanta, Allegri Ricardo Francisco

\begin{abstract}
Background: The diagnostic criteria for the semantic variant of primary progressive aphasia (svPPA) include the possible presence of acquired surface dyslexia or dysgraphia. Acquired surface dyslexia is characterized by a greater difficulty in reading irregular words with the production of regularization errors. On the other hand, acquired surface dysgraphia is characterized by difficulties in writing irregularly spelled words, which are stimuli that produce phonologically plausible errors. The identification of these patterns in Spanish has been subject to controversy due to the orthographic transparency of the language and its lack of irregular words. However, differences do exist between reading (completely transparent) and writing (considerably irregular) and thus, designing tests to identify acquired surface dysgraphia is a simple task, since there are irregular words for writing in Spanish. Nevertheless, few cases of acquired surface dysgraphia have been reported in Spanish-speaking patients. In addition, the identification of acquired surface dyslexia in Spanish-speaking patients requires the use of tasks other than reading irregular words. Aims: The aim of this paper is to report the reading and writing impairments of a Spanish-speaking patient with SVPPA, and show that it is possible to identify patterns of acquired surface dysgraphia and dyslexia in a transparent language. Methods and procedure: Single case study of a Spanishspeaking patient with svPPA. The tests administered were: writing to dictation of words (both regular and irregular) and nonwords, reading of words and nonwords (measuring accuracy and reaction times) and lexical decision with pseudohomophones. Outcomes and results: We found a regularity effect in the writing to dictation task with the generation of several phonologically plausible errors, a loss of lexical advantage in reading latencies although reading accuracy was conserved, and a pseudohomophone disadvantage effect in the lexical decision task; thus, resulting in a profile consistent with the patterns of acquired surface dysgraphia and dyslexia. Conclusions: The results obtained indicate it is possible to identify these patterns when the characteristics of the language are taken into consideration in the selection of tests to be administered. Therefore, it is possible to test for all the established criteria for svPPA in Spanishspeaking patients.
\end{abstract}

Palabras clave

Primary progressive aphasia, semantic dementia, acquired surface dyslexia, acquired surface dysgraphia, Spanish-speaking patient. 